\title{
TIPIFICACIÓN INTEGRAL DE SISTEMAS CAPRINOS DE LA PROVINCIA DE SANTA ELENA, ECUADOR
}

\section{INTEGRAL TYPIFICATION OF GOAT SYSTEMS OF SANTA ELENA PROVINCE, ECUADOR}

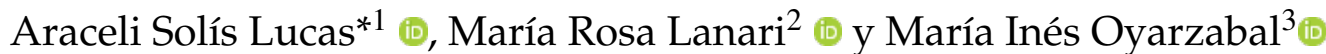

\author{
${ }^{1}$ Facultad de Ciencias Agrarias. Universidad Estatal Península de Santa Elena. Becaria de la Secretaria de Educación Superior, \\ Ciencia, Tecnología e Innovación, SENESCYT. La Libertad, Ecuador. \\ 2 Instituto Nacional de Tecnología Agropecuaria (INTA). Estación Experimental Agropecuaria (EEA). Grenville Morris, 8400. \\ Bariloche, Argentina. \\ ${ }^{3}$ Facultad de Ciencias Veterinarias. CIC. Universidad Nacional de Rosario. 2170 Casilda. Argentina. \\ *Autor para correspondencia: solisluara@hotmail.com
}

Manuscrito recibido el 27 de abril de 2019. Aceptado, tras revisión, el 4 de diciembre de 2019. Publicado el 1 de marzo de 2020.

\begin{abstract}
Resumen
Como paso previo para proponer estrategias de desarrollo en sistemas de producción caprina (SPC) de la provincia de Santa Elena, Ecuador, se propuso tipificar los sistemas mediante indicadores socio-económicos, productivos y ambientales. Se encuestó a 172 productores sobre composición familiar y participación en el trabajo, tecnología adoptada, comunicaciones, composición del hato, existencias ganaderas, uso del suelo, ingresos, infraestructura y servicios básicos, acceso a las explotaciones, asistencia técnica y capacitación, trabajo e ingresos extraprediales, manejo e instalaciones, fuente de agua y suplemento alimenticio, aspecto reproductivo, dificultades en la producción de caprinos, comercialización de caprinos. Se redujo el número de variables mediante análisis de componentes principales (ACP) y pruebas de $\chi^{2}$, para las variables cuantitativas y cualitativas, respectivamente. Con las CP que explicaron el $80 \%$ de la variabilidad se llevó a cabo un análisis de conglomerados (AC) que dividió a los establecimientos en 7 grupos. Mediante un análisis de correspondencias múltiples (ACM) se identificaron asociaciones entre grupos y modalidades de las variables cualitativas. Las variables que más aportaron a la diferenciación de los grupos de SP estaban relacionadas con la producción caprina, el tamaño de la familia, las producciones avícola, agrícola, porcina y bovina, la participación en el trabajo de la familia, los ingresos por beneficios sociales, el tamaño de la finca y la tecnología adoptada. Dos grupos eran mixtos; uno con mayor actividad porcina; dos se caracterizaron por la cría de cabras para el consumo cuyos ingresos provenían de fuentes externas y dos grupos se definieron por la edad de sus productores.
\end{abstract}

Palabras clave: Indicadores económicos, indicadores productivos, indicadores socio-culturales, sistemas de producción, cabras. 


\begin{abstract}
As a preliminary step to propose development strategies in the goat productive systems (SPC) of the Santa Elena province, Ecuador, the SPC were typified by means of socio-economic, productive and environmental indicators. A sample of 172 producers was surveyed on family composition and participation in work, adopted technology, communications, composition of the herd, livestock stock, land use, income, infrastructure and basic services, access to farms, technical assistance and training, off-farm work and income, management and facilities, water sources and food supplement, reproductive aspects, difficulties in the production of goats, marketing of goats. The number of proposed variables was reduced by principal component analysis (PCA) and $\chi^{2}$ tests for quantitative and qualitative variables, respectively. Based on the main components that explained $80 \%$ of the variability, a conglomerate analysis (CA) was carried out, resulting in the division of the SPC into 7 groups. Through a multiple correspondence analysis (MCA), associations between groups and modalities of qualitative variables were identified. The variables that contributed most to the differentiation of SPC groups were related to goat production, family size, poultry, agricultural, pig and bovine production, participation in family work, income from social benefits, the size of the farm and the technology adopted. Two groups were mixed, one with a predominance of livestock activity and the other with agricultural activity; a group with greater swine activity; two groups were characterized by the breeding of goats for consumption, whose income came from external sources; and two groups were defined by the age of their producers.
\end{abstract}

Keywords: Economic indicators, productive indicators, socio-cultural indicators, production systems, goats.

Forma sugerida de citar: Solís Lucas, A., Lanari, M.R. y Oyarzabal, M.I. (2020). Tipificación integral de sistemas caprinos de la provincia de Santa Elena, Ecuador. La Granja: Revista de Ciencias de la Vida. Vol. 31(1):82-95. http://doi.org/10.17163/lgr.n31.2020.06.

IDs Orcid:

Ligia Araceli Solís Lucas: http:/ / orcid.org/ 0000-0002-4428-3888

María Rosa Lanari: http:/ / orcid.org/0000-0002-2186-601X

María Inés Oyarzabal: http:/ / orcid.org/0000-0001-5429-5789 


\section{Introducción}

El conocimiento de la realidad de los sistemas productivos es necesario para proponer soluciones frente a situaciones críticas, implementar planes de mejoramiento genético, evaluar el impacto ambiental de la ganadería, entre otras. La identificación de las fortalezas y debilidades permite proponer estrategias que debieran ser específicas para cada tipo de unidades de producción si existiera diversidad entre los sistemas de una región determinada $(\mathrm{Ca}-$ brera y col., 2004; Dufumier, 1990).

Se recomienda que las alternativas de desarrollo para los sistemas productivos de subsistencia se propongan conjuntamente con los productores (Macías, 2015). Se trata de estimular el profundo interés personal por el cuidado de los animales y los cultivos; ofrecer más productos para la venta mediante un mejor uso de lo que está disponible localmente (recursos biológicos, vegetación autóctona, ganado, etc.); introducir conocimiento y tecnología junto con la valoración de las competencias, conocimientos y experiencias existentes; colaborar con los productores para que realicen mejor lo que ellos hacen (Hodges y col., 2014). Por otro lado, los sistemas de subsistencia albergan valiosos recursos zoogenéticos que es necesario conservar debido a que han evolucionado en su adaptación a ambientes adversos y existen situaciones en las que se está perdiendo biodiversidad genética (Núñez-Domínguez y col., 2016; Dorji, Tamang y Dorji, 2017).

A partir del análisis del estado inicial y de la caracterización de los sistemas se puede identificar y tipificar grupos diferenciados. Se han propuesto distintos métodos para llevar a cabo la estratificación basada en un amplio rango de aspectos ambientales, productivos, económicos y sociales, que responden a diferentes objetivos. Otra forma de estratificación de los sistemas consiste en la aplicación de métodos estadísticos de agrupamiento (Robinson y col., 2011).

Los sistemas productivos caprinos (SPC) de la provincia de Santa Elena (PSE), Ecuador, son sistemas de subsistencia de bajos recursos. Santa Elena posee el mayor porcentaje de caprinos a nivel de la región costa $(53 \%)$ y el 7,2\% del total de las existencias del país (Sistema Nacional de Información, 2018). Como paso previo al diseño de planes de desarrollo, se propuso probar si existe heterogeneidad entre los sistemas caprinos de la provincia. Se llevó a cabo un proceso que incluyó: relevamiento de los establecimientos, encuesta a los productores y tipificación de los sistemas productivos predominantes, mediante el uso de indicadores socioculturales, productivos y económicos.

\section{Materiales y Métodos}

Santa Elena es una provincia de la costa de la República de Ecuador, ubicada al suroeste del litoral ecuatoriano. Políticamente está dividida en tres cantones: La Libertad, Salinas y Santa Elena (3.668,9 $\mathrm{km}^{2}$ ). Este último es el cantón más grande de la provincia, con una alta población rural. Tiene 67 comunas registradas en la Dirección Provincial Agropecuaria de Santa Elena (Ministerio de Agricultura y Ganadería, 2018).

A partir de información oral recibida de los comuneros y del Ministerio de Agricultura y Ganadería (MAG), se ubicó a 586 sistemas productivos que poseían cabras, con un total estimado de 11 977 cabezas, en las 3 zonas climáticas en las que se está dividida la provincia. Dentro de cada zona, se realizó una distribución de frecuencias del número de productores caprinos según tamaño del hato. Se estimó el número total de establecimientos a muestrear mediante la aplicación de la fórmula para muestra finita (FAO, 2012). Una vez definido el tamaño muestral total, se realizó un muestreo estratificado por zona y tamaño del hato mediante asignación proporcional.

Se diseñó una encuesta estructurada para realizar a los productores, tomando como referencia a las utilizadas por Falagan, 1988, Bedotti, 2000 y Lanari, 2004, incluyendo aspectos socio-culturales, productivos y económicos (Tablas 1, 2 y 3). Para las variables cuantitativas, se probó si existían diferencias entre las zonas agroecológicas mediante un análisis de la varianza multivariado (MANOVA). Con el propósito de disminuir el número de variables: se estimó el grado de asociación entre las 27 variables cuantitativas para definir la exclusión de aquellas variables que fueran indicativas de la misma característica $(r>0,70)$ y se realizó un análisis de componentes principales (ACP) (Peña, 2002), eligiéndose las componentes que explicaran al menos el $80 \%$ de la variabilidad total. 
Tabla 1. Variables cuantitativas relacionadas con los aspectos socio-cultural, productivo y económico

\begin{tabular}{lll}
\hline \multicolumn{1}{c}{ Tipo de información } & \multicolumn{1}{c}{ Variables } & ED \\
& Edad del productor en años & TP \\
Composición familiar & Nro. personas integrantes de la familia que viven en la misma casa & PD \\
y participación en el trabajo & Nro. personas que trabajan exclusivamente dentro de la finca & PF \\
& Nro. personas que trabajan exclusivamente fuera de la finca & PDF \\
& Nro. personas que trabajan dentro y fuera de la finca & GCR \\
\multirow{2}{*}{ Tecnología adoptada } & Grado de conocimiento del ciclo reproductivo & GCS \\
\hline Comunicaciones & Grado de control sanitario & DL \\
\hline \multirow{4}{*}{$\begin{array}{l}\text { Composición del hato } \\
\text { (número de cabezas por }\end{array}$} & Distancia a la localidad con la que el productor más se comunica (km) & CHVA \\
categoría) & Hembras chivas & CBLA \\
& Hembras cabrillas & CBTA \\
& Machos chivos & CHVO \\
& Machos chivitos & CHTO \\
& Machos cabritos & CBTO \\
\hline \multirow{2}{*}{ Existencias ganaderas } & Caprinas & EC \\
& Bovinas & EB \\
& Porcinas & EP \\
& Avícolas & EA \\
\hline \multirow{2}{*}{ Uso del suelo } & Hectáreas sembradas & HaS \\
& Superficie de la finca en hectáreas & HaF \\
\hline & Por venta de cultivos agrícolas & \$IAG \\
Ingresos en \$ en el & Por venta bovina & \$IB \\
último año & Por venta caprina & \$IC \\
& Por venta porcina & \$IP \\
& Por venta avícola & \$IA \\
& Por otras fuentes extraprediales & \$IEX \\
& Por beneficios sociales & \$IBS \\
\hline
\end{tabular}

Con las componentes principales seleccionadas, mediante un análisis de conglomerados (AC) (Dallas, 2000), se clasificaron los sistemas productivos en grupos homogéneos; se aplicaron: el método jerárquico, la distancia euclídea y el método de Ward (Ward, 1963). Los perfiles de cada grupo (G) se describieron mediante los promedios estandarizados de todas las variables y se los representó en un gráfico radial.

Para las 77 variables cualitativas, se estimaron las frecuencias relativas para cada uno de los grupos de productores resultantes del AC y las frecuencias totales. Con el objetivo de seleccionar las variables para las cuales existirían diferencias significativas entre los grupos se realizaron pruebas $\chi^{2}$ de Pearson $(p<0,05)$. Con las variables seleccionadas se realizó un análisis de correspondencias múltiples (ACM) para identificar las asociaciones de mayor peso entre las modalidades de las variables cualitativas (Greenacre, 1984) y los grupos de productores. Los cálculos estadísticos se realizaron con el programa Infostat profesional (Di Rienzo y col., 2008).

\section{Resultados}

A partir de la información recibida sobre el número total de productores por zona, se los distribuyó según el tamaño del hato y se estimó la muestra en 229. La asignación proporcional por zona y tamaño del hato se detalla en la Tabla 4. De estos, el $22 \%$ no fue encuestado por diversas causas: información duplicada, ausencia del productor al momento de la visita, abandono de la actividad, etc. 
Tabla 2. Variables cualitativas relacionadas con los aspectos socio-cultural y económico

\begin{tabular}{|c|c|c|c|c|}
\hline $\begin{array}{l}\text { Datos el } \\
\text { productor }\end{array}$ & $\begin{array}{l}\text { Infraestructura, servicios } \\
\text { básicos }\end{array}$ & $\begin{array}{l}\text { Acceso a las } \\
\text { explotaciones }\end{array}$ & Organizacional & Laboral \\
\hline $\begin{array}{l}\text {-Persona } \\
\text { entrevistada } \\
\text { (PEN) } \\
\text {-Sexo (SEX) } \\
\text {-Estado civil } \\
\text { (ECI) } \\
\text {-Nivel de } \\
\text { estudios } \\
\text { (EDU) } \\
\text {-Seguro social } \\
\text { (SEG) } \\
\text {-Ayudante } \\
\text { (AYU) } \\
\text {-Sucesor } \\
\text { (SUC) }\end{array}$ & $\begin{array}{l}\text {-Tenencia de la tierra (TTI) } \\
\text {-Pertenencia de la vivienda } \\
\text { (PVI) } \\
\text {-Tipo de vivienda (TVI) } \\
\text {-Material de construcción de la } \\
\text { vivienda (MCV) } \\
\text {-Estado de la vivienda (EVI) } \\
\text {-Servicio de alumbrado (SAL) } \\
\text {-Dotación agua potable (DAP) } \\
\text {-Medio de acceso agua (MAA) } \\
\text {-Uso de energía para preparar } \\
\text { los alimentos (UEN) } \\
\text {-Servicio de alcantarillado } \\
\text { (ALC) } \\
\text {-Medio de alcantarillado (MAL) } \\
\text {-Acceso a la tecnología (ACT) }\end{array}$ & $\begin{array}{l}\text {-Localidad con la } \\
\text { que más se } \\
\text { comunican (LCO) } \\
\text {-Vías de acceso } \\
\text { (VAC) } \\
\text {-Tipo de carretera } \\
\text { o camino (TCA) } \\
\text {-Estado de la } \\
\text { carretera (ECA) } \\
\text {-Medio de } \\
\text { transporte (MTR) }\end{array}$ & $\begin{array}{l}\text {-Participa en alguna } \\
\text { organización (POrg) } \\
\text {-Organización en la } \\
\text { que participa (OPA) } \\
\text {-Recibe asistencia } \\
\text { técnica (RAT) } \\
\text {-Institución de la que } \\
\text { recibe asistencia } \\
\text { técnica (IRA) } \\
\text {-Tipo de institución } \\
\text { (TIS) } \\
\text {-Aplica técnicas } \\
\text { (ATE) } \\
\text {-Observó resultados } \\
\text { (ORE) } \\
\text {-Recibe capacitación } \\
\text { (RCA) } \\
\text {-Interés en recibir } \\
\text { capacitación (IRC) }\end{array}$ & $\begin{array}{l}\text {-Empleo } \\
\text { extrapredial } \\
\text { (EEE) } \\
\text {-Tipo de entidad } \\
\text { en la que trabaja } \\
\text { (TET) } \\
\text {-Tipo de empleo } \\
\text { (TEM) } \\
\text {-Tipo de labor que } \\
\text { realiza (TLR) } \\
\text {-Posee otras } \\
\text { fuentes } \\
\text { extraprediales } \\
\text { (OFE) } \\
\text {-Procedencia de } \\
\text { los ingresos de } \\
\text { otras fuentes } \\
\text { extraprediales } \\
\text { (IOF) }\end{array}$ \\
\hline
\end{tabular}

No se encuestó a ninguno de los dos productores pertenecientes a la zona semi-húmeda debido a la inaccesibilidad al lugar al momento del relevamiento. Se relevaron en total 172 establecimientos, 69 en la zona seca y 103 en la zona semiárida.

Los resultados de la aplicación del MANOVA no evidenciaron diferencias significativas entre las dos zonas agroecológicas para las variables cuantitativas (Tabla 1). Por lo cual, en adelante, los análisis se presentan tomando a las dos zonas en conjunto.

De las 27 variables cuantitativas originales, se excluyeron: a) las variables relacionadas con la composición del hato dado que estaban altamente correlacionadas con EC; b) distancia a la localidad con la que el productor más se comunica por no aportar a la variabilidad total en el primer análisis ACP. Con las 20 variables restantes se realizó un segundo ACP. Las primeras 12 componentes explicaron el $87 \%$ de la variabilidad total. En base a los coeficientes de mayor peso de cada componente y a su signo se interpretaron como se describe en la Tabla 5.

El análisis de conglomerados realizado con las 12 primeras componentes principales dividió a los establecimientos productivos en 7 grupos distri- buidos en ambas zonas agroecológicas. Más de la mitad de los establecimientos se encontraban en los G5 $(23,8 \%)$ y G6 $(30,2 \%)$. El menor porcentaje lo compartieron los G3 (2,3\%) y G7 (4,07\%). A los G1, G2 y G4 le correspondieron $14 \%, 17,4 \%$ y $8,1 \%$ respectivamente.

La Tabla 6 muestra los promedios de las 20 variables para cada uno de los 7 grupos y el promedio general. En la Figura 1 se representan los promedios estandarizados de las variables mediante un gráfico radial general. El círculo negro indica el valor cero o medio de cada variable estandarizada. Por ejemplo, el G3 tiene valores por encima del promedio en cuanto al trabajo familiar dentro de la finca (PD) y a los ingresos caprinos ( $\$ \mathrm{IC})$, aunque las existencias caprinas están por debajo de la media; es el grupo con mayor producción avícola.

Se excluyó del análisis a aquellas variables cualitativas que no aportaban variabilidad dentro de los grupos y tenían una frecuencia relativa para una categoría mayor al 95\% (PEN, TTI, PVI, TVI, SAL, ALC, MAL, VAC, TEM, IOF, ICA, CEC, TI, SCT, CDC, DOB, VRE, LCO, FPA). Por ejemplo, para SAL, el $100 \%$ de los productores expresaron tener servicio de alumbrado eléctrico. Las pruebas $\chi^{2}$ 
para las 58 variables restantes evidenciaron diferencias significativas $(p<0,05)$ entre los siete grupos MCP, OC, MPR).

Tabla 3. Variables cualitativas relacionadas con el aspecto productivo caprino

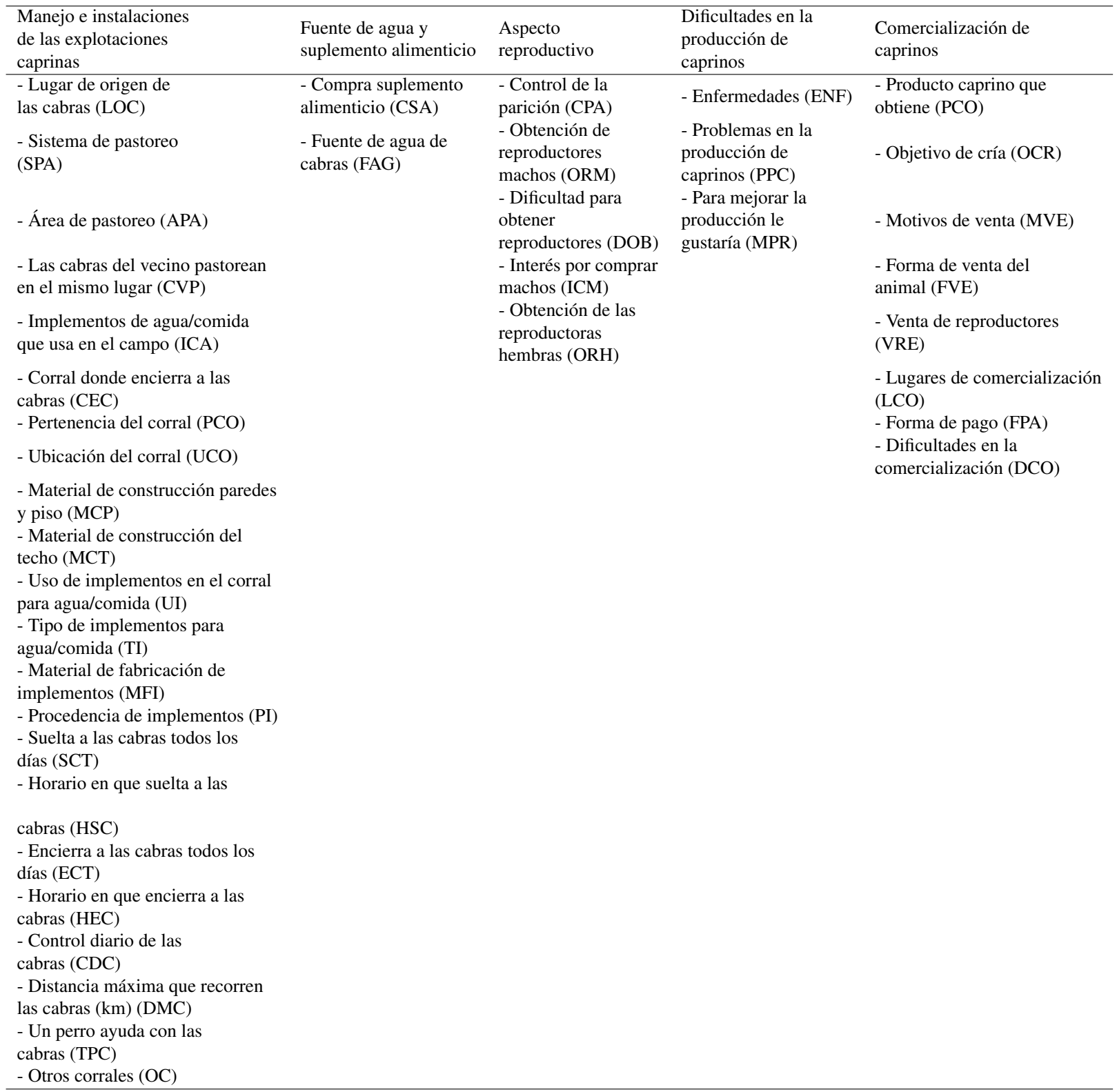

El ACM mostró que en la primera dimensión pesó el recibir asistencia técnica y capacitación (RATsi y RCAsi), en la segunda el no acceso a la tecnología (ACTno).Estas primeras dos dimensiones explicaron el $37 \%$ de las variabilidad de las variables socioculturales y de manejo. La tercera, cuarto y quin- ta dimensiones estuvieron relacionadas con el interés de recibir o no capacitación, la sexta con la finca como área de pastoreo de las cabras. 


\subsection{Descripción general de los sistemas productivos de la península de Santa Elena}

La mayoría de los dueños de los hatos caprinos son varones, con estudios de primaria (61\%) y con seguro social campesino, cuyos ayudantes en el manejo de las cabras es un familiar, sus hijos continuarían con la cría de cabras.

Los productores participan de las reuniones $\mathrm{CO}^{-}$ munales y solo un tercio afirmó recibir asistencia técnica, la mayoría de los beneficiarios fueron aquellos productores que participaron de proyectos estatales y que introdujeron cabras de otros lugares en las dos últimas décadas. Existe un gran interés de los pequeños productores de la PSE por mejorar la producción caprina (94\%), a pesar de que los mayores ingresos actuales no provienen de la producción caprina sino de otras fuentes externas. Tienen ingresos extraprediales, con empleos en empresas privadas y de forma temporaria o reciben el bono de desarrollo humano (BDH).

Los establecimientos caprinos de la PSE se encuentran en zonas comunales y con derecho de posesión con viviendas en estado regular. La totalidad de las fincas posee alumbrado eléctrico, sin alcantarillado, con acceso al agua a través de la red pública (57\%). La provincia cuenta, con vías de acceso, con carreteras no asfaltadas, en mal estado, no existe transporte público.

El manejo de las cabras es a libre pastoreo, no utilizan implementos para agua y comida en el campo. Los productores poseen corrales propios, ubicados cerca de la casa, con piso de tierra y techo. En general, los productores sueltan a las cabras y las encierran cuando regresan; en invierno, los animales pueden permanecer hasta 3 meses en el campo. Las cabras obtienen su alimentación en el campo, si los productores cultivan maíz, las cabras aprovechan los rastrojos del cultivo y en algunos casos reciben restos de alimentos en las temporadas más secas. La suplementación no es una práctica común debido a los costos que implica. El consumo de agua de los caprinos proviene de fuentes naturales, no así en la época seca, en la que se ven obligados a buscar otras fuentes de agua (potable). Los machos permanecen con las hembras en el hato, hasta la venta después de los 8 meses de edad.

No se utilizan prácticas como la castración. La mayoría de los reproductores machos son de propia producción. Se detecta a las cabras cuando están por parir, se separan y retienen en el corral. El grado de control sanitario (GCS) es muy bajo, con algún tipo de problema sanitario.

El producto que se obtiene es la carne, muy pocos obtienen leche o aprovechan el estiércol como abono; solo el $24 \%$ cría exclusivamente para la venta, el $16 \%$ los vende para el sustento familiar y el $84 \%$ vende sus animales en emergencias familiares. La venta de las cabras se realiza en pie, en las casas o fincas y solo el $5 \%$ se vende al cantón comercial de la provincia y a otras localidades, recibiendo el pago de contado. Solo el $6 \%$ de los productores vende en raras ocasiones algún macho como reproductor.

\subsection{Características específicas de los gru- pos de sistemas productivos caprinos de la provincia de Santa Elena (Tabla 6 y Figura 1):}

Grupo 1: constituido por las familias numerosas, con el promedio más alto de personas que trabajan fuera de la finca. Poseen muy bajo conocimiento del ciclo reproductivo (CR) y control sanitario (CS), recibieron muy escasa asistencia técnica y capacitación, tienen acceso al uso de tecnología. Cuentan con las existencias ganaderas más bajas, incluidos los caprinos, y bajo número de hectáreas sembradas, están entre las fincas de menor superficie. Tienen altos ingresos extraprediales e ingresos por beneficios sociales superiores a la media.

Grupo 2: productores jóvenes, con familias de tamaño intermedio, sus integrantes dedican al menos parte de su jornada laboral al trabajo en la finca. Tienen el mayor grado de conocimiento del CR y CS, la mitad de ellos recibieron asistencia técnica y/o capacitación. Las fincas tienen la mayor superficie total y sembrada. Las existencias ganaderas están por debajo del promedio general, el $71 \%$ corresponde a existencias caprinas. Tienen el mayor ingreso total y el mayor ingreso por venta de productos agrícolas, están entre los de más altos ingresos caprinos e ingresos externos. 
Tabla 4. Número total de productores, cabras y productores a encuestar por zonas según tamaño del hato

\begin{tabular}{ccccccc}
\hline Tamaño del hato & \multicolumn{2}{l}{ Número total de productores } & \multicolumn{3}{c}{ Número de productores a encuestar } \\
& Zona & Zona & Zona & Zona & Zona & Zona \\
& semiárida & seca & semihúmeda & semiárida & seca & semihúmeda \\
\hline $5-25$ & 290 & 159 & 2 & 113 & 62 & 1 \\
$26-45$ & 54 & 24 & & 21 & 9 & \\
$46-65$ & 15 & 9 & & 6 & 4 & \\
$66-85$ & 7 & 5 & & 3 & 2 & \\
86 y más & 13 & 8 & & 5 & 3 & \\
\hline Total productores/zona & 379 & 205 & 2 & 148 & 80 & 1 \\
\hline Total cabras/zona & 7.363 & 4.594 & 20 & & & \\
\hline
\end{tabular}

Fuente: MAG, FEDECOMSE, Asociación de Capricultores, Casas Comunales, productores (Información oral)

Tabla 5. Interpretación de las doce primeras componentes principales

\begin{tabular}{|c|c|c|c|c|}
\hline Componente & \multicolumn{2}{|l|}{ Interpretación } & $\begin{array}{c}\text { Variabilidad } \\
\text { explicada (\%) }\end{array}$ & $\begin{array}{c}\text { Acumulado } \\
\%\end{array}$ \\
\hline$Y_{1}$ & \multicolumn{2}{|l|}{ Producción caprina } & 14 & 14 \\
\hline$Y_{2}$ & \multicolumn{2}{|l|}{ Tamańo de la familia } & 12 & 26 \\
\hline$Y_{3}$ & \multicolumn{2}{|l|}{ Producción avícola } & 10 & 36 \\
\hline$Y_{4}$ & \multicolumn{2}{|c|}{ Producción agrícola vs producción porcina } & 9 & 45 \\
\hline$Y_{5}$ & \multicolumn{2}{|c|}{ Producción bovina } & 8 & 54 \\
\hline$Y_{6}$ & \multirow{3}{*}{ Participación en el trabajo: } & PD & 6 & 60 \\
\hline$Y_{7}$ & & PDF & 5 & 65 \\
\hline$Y_{8}$ & & $\mathrm{PF}$ & 5 & 70 \\
\hline$Y_{9}$ & \multicolumn{2}{|c|}{ Ingresos por beneficios sociales } & 5 & 75 \\
\hline$Y_{10}$ & \multicolumn{2}{|l|}{ Tamańo de la finca } & 4 & 79 \\
\hline$Y_{11}$ & \multirow{2}{*}{ Tecnología adoptada: } & GCR & 4 & 83 \\
\hline$Y_{12}$ & & GCS & 3 & 87 \\
\hline
\end{tabular}

Grupo 3: la edad de los productores es cercana a la media, con familias numerosas que trabajan principalmente dentro de la finca. Tienen buen conocimiento del CR y escaso CS, todos los establecimientos del grupo recibieron asistencia técnica y capacitación. Sus existencias avícolas son las más altas y el número de hectáreas sembradas es de los más altos. Tienen altos ingresos por venta de productos agrícolas y caprinos, y los más altos por ventas avícolas. Tienen bajos ingresos externos.

Grupo 4: la edad de los productores está en el promedio, el tamaño de las familias es de los más pequeños, sus integrantes trabajan dentro y fuera de la finca. El grado de conocimiento del CR está por encima del promedio, a pesar de que recibieron muy escasa asistencia técnica y capacitación.
Poseen las más altas existencias porcinas y existencias caprinas medias, con superficies intermedias de hectáreas sembradas. Tienen los ingresos por venta de porcinos más elevados y los ingresos extraprediales intermedios.

Grupo 5: son los productores de mayor edad, con el más bajo número de integrantes de la familia, que trabajan fundamentalmente dentro de la finca. El grado de conocimiento del CR es cercano a la media, pero el CS es de los más bajos. Recibieron escasa asistencia técnica y capacitación. Son los que tienen menor acceso a la tecnología. Las existencias pecuarias son bajas, pero tienen existencias caprinas medias. El número de hectáreas sembradas es el más bajo, es el segundo grupo con mayor tamaño de la finca. Los ingresos por beneficios sociales 
(BDH) son los más altos y los ingresos extraprediales son los más bajos.

Tabla 6. Promedios por grupos de establecimientos

\begin{tabular}{lcccccccc}
\hline Grupos & $\mathbf{1}$ & $\mathbf{2}$ & $\mathbf{3}$ & $\mathbf{4}$ & $\mathbf{5}$ & $\mathbf{6}$ & $\mathbf{7}$ & $\begin{array}{c}\text { Promedio } \\
\text { general }\end{array}$ \\
\hline ED & 55,1 & 49,5 & 57,7 & 57,3 & 71,6 & 46,4 & 55,3 & 56,1 \\
TP & 6,75 & 4,97 & 5,00 & 3,71 & 2,80 & 4,81 & 3,43 & 4,50 \\
PD & 1,68 & 1,65 & 3,13 & 1,52 & 1,43 & 2,12 & 1,57 & 1,87 \\
PF & 1,14 & 0,08 & 0,00 & 0,39 & 0,02 & 0,02 & 0,14 & 0,26 \\
PDF & 0,78 & 0,66 & 0,35 & 0,40 & 0,24 & 0,39 & 0,50 & 0,47 \\
GCR & 2,83 & 5,20 & 4,25 & 4,50 & 4,02 & 4,12 & 5,00 & 4,27 \\
GCS & 0,88 & 2,90 & 1,50 & 1,71 & 1,49 & 1,90 & 2,57 & 1,85 \\
EC & 11,5 & 24,6 & 21,0 & 24,1 & 25,8 & 16,1 & 54,6 & 25,40 \\
EB & 1,04 & 4,07 & 0,25 & 1,79 & 1,83 & 0,48 & 31,3 & 5,82 \\
EP & 0,42 & 1,23 & 3,50 & 10,6 & 0,49 & 0,65 & 4,29 & 3,02 \\
EA & 3,13 & 4,57 & 76,2 & 8,07 & 0,61 & 2,23 & 3,86 & 14,10 \\
HaS & 0,96 & 2,89 & 1,63 & 1,00 & 0,51 & 0,80 & 0,71 & 1,210 \\
HaF & 3,54 & 8,48 & 5,00 & 4,54 & 6,76 & 2,01 & 7,71 & 5,43 \\
\$IAG & 412 & 1941 & 1147 & 277 & 110 & 333 & 1091 & 759 \\
\$IB & 25,4 & 52,7 & 0,00 & 42,9 & 7,32 & 5,00 & 607 & 105 \\
\$IC & 41,2 & 225 & 355 & 164 & 175 & 84,4 & 355 & 199 \\
\$IP & 0,00 & 12,3 & 50,0 & 396 & 0,00 & 12,9 & 146 & 88,2 \\
\$IA & 4,08 & 9,33 & 540 & 22 & 0,00 & 3,85 & 0,00 & 82,7 \\
\$IEX & 2316 & 2222 & 690 & 2039 & 472 & 2688 & 1522 & 1707 \\
\$IBS & 551 & 481 & 450 & 429 & 759 & 404 & 429 & 500 \\
Iing & 3350 & 4945 & 3232 & 3370 & 1524 & 3531 & 4150 & 3443 \\
IEx & 16,1 & 34,4 & 101 & 44,6 & 28,8 & 19,4 & 94 & 48,3 \\
\hline
\end{tabular}

ED: edad del productor, TP: número personas integrantes de la familia que viven en la misma casa, PD: número personas que trabajan exclusivamente dentro de la finca, PF: número personas que trabajan exclusivamente fuera de la finca, PDF: nro. personas que trabajan dentro y fuera de la finca, GCR: grado de conocimiento del ciclo reproductivo, GCS: grado de control sanitario, EC: existencias caprinas, EB: existencias bovinas, EP: existencias porcinas, EA: existencias avícolas, HaS: hectáreas sembradas, HaF: superficie de la finca en hectáreas, \$IAG: total venta de cultivos agrícolas, \$IB: total venta bovina, \$IC: total venta caprina, \$IP: total venta porcina, \$IA: total venta avícola, \$IEX: ingresos por otras fuentes extraprediales, \$IBS: ingresos por beneficios sociales, $\sum$ Ing: ingresos totales, $\sum \mathbf{E x}$ : existencias totales.

Grupo 6: son los productores más jóvenes, con tamaño de familia intermedio y alto número de personas que trabajan dentro de la finca. El conocimiento del CR y CS están cercanos a la media, recibieron poca asistencia técnica o capacitación. Son las fincas de mayor superficie, la superficie sembrada es de las más bajas y tienen bajas existencias ganaderas. Poseen los ingresos extraprediales más altos y los más bajos por beneficios sociales.

Grupo 7: son productores de edad media, familias pequeñas, cuyos integrantes trabajan dentro y fuera de la finca. Tienen alto grado de conocimiento del CR y muy alto CS, tienen acceso a la tecnología. Recibieron asistencia técnica y capacitación. Es el segundo grupo en existencias ganaderas, tienen las más altas existencias caprinas y bovinas, y existencias porcinas intermedias, están dentro de las fincas con mayor superficie. Es el grupo con mayores ingresos por venta de bovinos y caprinos, y tiene uno de los ingresos más altos por venta de porcinos. Es el segundo grupo con altos ingresos totales. 


\section{Discusión}

La tipificación de los sistemas productivos caprinos de la PSE permitió definir características comunes a los 172 establecimientos e identificar la heterogeneidad presente clasificándolos en 7 grupos, distribuidos de manera homogénea por toda la provincia. Se encontraron 2 grupos mixtos, uno con predominancia de la actividad pecuaria (G3) y otro de la ac- tividad agrícola (G2); un grupo dedicado más a la actividad pecuaria porcina (G4); 2 grupos definidos por la cría de cabras para el consumo cuyos ingresos provienen de fuentes externas (G1 y G6); y otros 2 grupos definidos por la edad de sus productores, cuyos ingresos provienen para el grupo de mayor edad de los beneficios sociales (G5) y para el grupo más joven de ingresos externos (G6).

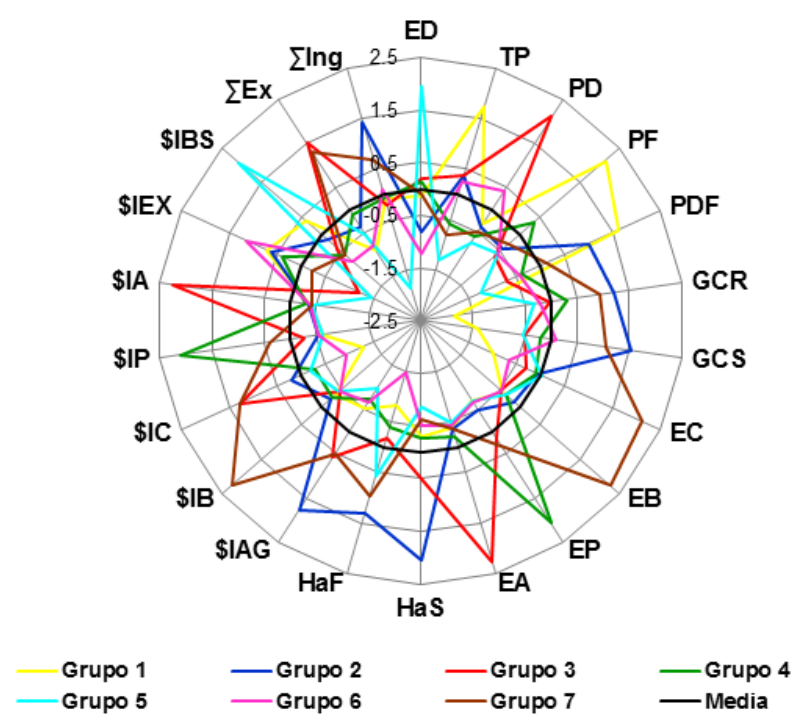

Figura 1. Perfiles de los siete grupos de sistemas productivos.

En otras regiones se utilizaron variables similares para tipificar establecimientos (Coronel de Renolfi, M. and Ortuño, S., 2005; Costa y col., 2008; Garcia y col., 2010; Hernández y col., 2011; Gómez, 2013; Hernández y col., 2013; Chivangulula y col., 2014; Umunna, Olafadehan y Arowona, 2014; Barboza, 2018). En este trabajo se incluyeron, además, los ingresos con el propósito de identificar diferentes estrategias de subsistencia.

Los establecimientos caprinos de la PSE están asentados en el área rural, en terrenos comunales con derecho de posesión. En otros países se encontraron otras formas de tenencia de la tierra: propiedad, comodato, ocupantes o localizados en tierras fiscales (Hernández y col., 2011; Vargas y col., 2014; Torres, 2014; Bedotti, 2000).

El nivel de educación es un aspecto de impor- tancia a resaltar si se quieren llevar adelante programas específicos que ayuden a mejorar la producción caprina. El 61\% de los productores de la PSE concluyeron la escuela primaria. Estos resultados difieren con lo reportado en otros países, donde más del $60 \%$ de los productores no tenían estudios (Serrano, 2010; Falagan, 1988; Luque, 2011; Bedotti y col., 2005; Gómez, 2013; Fikru y Gebeyew, 2015; Hagos y col., 2016).

La continuidad en la crianza de las cabras por parte de los hijos de los propietarios, manifestado en el $62 \%$ de las encuestas, es otro aspecto a resaltar pues deja abierta la posibilidad de proponer estrategias a más largo plazo. Este porcentaje es superior al $42 \%$ de la región de Murcia, España (Falagan, 1988), pero inferior al $80 \%$ o más de otros lugares (Bedotti, 2000; Luque, 2011; Gómez, 2013). 
El 97\% de los sistemas de la PSE son sistemas familiares, el propietario/a es quien se ocupa del cuidado de los caprinos, sin contratación de personal asalariado. Si bien la mayoría eran hombres (63\%), el 37\% de participación de las mujeres en la crianza caprina es muy superior al $20 \%$ de sistemas de México y Etiopía (Serrano, 2010; Tsegaye, 2009; Hagos y col., 2016). La presencia de la mujer en las actividades caprinas podría estar relacionada con el tipo de animal que se cría y con la habilidad para su manejo (Serrano, 2010).

De acuerdo a la clasificación de sistemas caprinos realizada por Devendra, 2015, los sistemas de la PSE se categorizan como extensivos y de libre pastoreo. No se encontró que los propietarios arrienden fincas o terrenos para el uso exclusivo de sus animales. Esto es similar a lo mencionado en Venezuela, Colombia, Perú y Etiopía (Valerio y col., 2010; Guerrero y col., 2012; Gómez, 2013; Hagos y col., 2016); pero diferente a otros lugares donde se arriendan terrenos para el pastoreo (Carné, Roig y Jordana, 2007).

El promedio general de cabras por hato en la PSE es 25. El bajo número se podría explicar por: a) el robo y la depredación; b) el objetivo de cría: solo el $25 \%$ de los productores vende sus animales para satisfacer parte de sus necesidades básicas, el resto los cría como medio de ahorro; c) la baja incorporación de tecnología y escasa asistencia técnica que genera bajos ingresos como en otras regiones (Costa y col., 2008; Valerio y col., 2010; Hernández y col., 2011; Navarro, Fernández y Perezgrovas, 2011; Gómez, 2013); d) la tenencia de la tierra ajena o comunal (Bellido y col., 2001).

Los ingresos anuales para los 7 grupos son variables, con un promedio mensual de \$287. Todos los grupos están muy por debajo de satisfacer las necesidades básicas mensuales de los productores dado que, de acuerdo al INEC, la canasta familiar básica mensual (CFB) al momento de este estudio tenía un valor de $\$ 425$.

Los sistemas caprinos de la PSE poseen características de sistemas rurales familiares, con una economía de subsistencia, con menos de 25 cabras por hato, con ingresos mensuales que no les permiten tener acceso a la canasta básica familiar y, por lo tanto, no cubren las necesidades básicas de los productores y sus familias. Se asimilan a la categoría de marginal propuesta por el Instituto Nacional de Estadística y Censos, 2010 y el Ministerio de Agricultura y Ganadería, 2018. Sin embargo, poseen conocimientos y experiencias adquiridas en el tiempo, en el manejo y cuidado de la biodiversidad. El manejo de esta biodiversidad adquirido a lo largo de años, al igual que un conjunto de conocimientos y prácticas, que no es fácil de describir, no debería desaparecer (Jarvis, Padoch y Cooper, 2011). Pues a pesar de la poca disponibilidad de activos y de los bajos niveles de productividad, la agricultura familiar es un importante proveedor de alimentos básicos de consumo popular (FAO/BID, 2007; Hodges y col., 2014).

\section{Conclusiones}

Por primera vez se ha tipificado a los sistemas productivos caprinos de la provincia de Santa Elena. Los resultados obtenidos en la presente investigación demuestran que existe heterogeneidad entre los sistemas caprinos santaelenenses y constituyen un aporte a para tener en cuenta cuando se quiera planificar opciones para potenciar su desarrollo.

\section{Agradecimientos}

A la Secretaría de Educación Superior, Ciencia, Tecnología e Innovación (SENESCYT) y la Universidad Estatal Península de Santa Elena (UPSE), sin cuyo apoyo económico, este trabajo no se hubiera realizado.

A los productores caprinos, quienes nos facilitaron toda la información y permitieron entrar en sus corrales.

\section{Referencias}

Barboza, M. (2018). «Caracterización de los sistemas de producción caprinos en la Región Huetar Norte de Costa Rica». Costa Rica: Universidad Nacional, Facultad de Ciencias de la Tierra y el Mar. Online: https://bit.ly/2GQIZ87. 
Bedotti, D. (2000). «Caracterización de los sistemas de producción caprina en el Oeste pampeano (Argentina)». Tesis Doctoral. Córdoba, España: Departamento de Producción Animal, Universidad de Córdoba. Online: https : / / bit . ly / 36ZEQZU.

Bedotti, D. y col. (2005). «Aspectos sociológicos de los sistemas de producción caprina en el Oeste Pampeano (Argentina)». En: Archivos de zootecnia 54.208, 599-608. Online: https: / / bit.ly / 2KULJFr.

Bellido, M. y col. (2001). "Sistemas extensivos de producción animal». En: Archivos de Zootecnia 50.192, 465-489. Online: https : / / bit . ly / 2GEdqOL.

Cabrera, D. y col. (2004). «Metodología para la caracterización y tipificación de sistemas ganaderos». En: Documentos de trabajo 1. Dpto. Producción Animal. Universidad de Córdoba 1, Online: https://bit.ly/2UU6TrW.

Carné, S., N. Roig y J. Jordana (2007). «La cabra Blanca de Rasquera: Caracterización estructural de las explotaciones». En: Archivos de Zootecnia 56.213, 43-54. Online: https:/ / bit.ly/2W49Wu5.

Chivangulula, M. y col. (2014). "Characterization of the livestock production cooperative systems in the municipality of Caála, Huambo province, Republico f Angola». En: Cuban Journal of Agricultural Science 48.2, 97. Online: https: / / bit.ly / 2WiOLGR.

Coronel de Renolfi, M. and Ortuño, S. (2005). «Tipificación de los sistemas productivos agropecuarios en el área de riego de Santiago del Estero, Argentina». En: Problemas del Desarrollo. Revista Latinoamericana de economía 36.140, 64-88. Online: https://bit.ly/2ZtrClb.

Costa, R. y col. (2008). «Caracterização do sistema de produção caprino e ovino na região semiárida do estado da Paraíba, Brasil». En: Archivos de Zootecnia 57.218, 195-205. Online: https:/ / bit. ly/2L05DPo.

Dallas, E. (2000). Métodos multivariados aplicados al análisis de datos. México: International Thomson Editores.

Devendra, C. (2015). «Dynamics of Goat Meat Production in Extensive Systems in Asia: Improvement of Productivity and Transformation of Livelihoods». En: Agrotechnoly 4.1, 131. Online: https://bit.ly/38Z81xP.
Di Rienzo, J. y col. (2008). InfoStat, versión 2008, Grupo InfoStat. Ed. por Argentina FCA Universidad Nacional de Córdoba.

Dorji, J., S. Tamang y T. Y. Dorji (2017). «Decline of Jakar sheep population in pastoral communities of Bhutan: A consequence of diminishing utility, alternate income opportunities and increasing challenges». En: Policy and Practice 7.4, Online: https:/ / bit.ly/2EEVyl9.

Dufumier, M. (1990). «Importancia de la tipología de unidades de producción agrícolas en el análisis de diagnóstico de realidades agrarias». En: Tipificación de sistemas de producción agrícola. Ed. por Germán Escobar y Julio Berdegué. Santiago de Chile: Red Internacional de Metodología de Investigación de Sistemas de Producción - RIMISP, 63-81. Online: https://bit.ly/392KjAV.

FAO (2012). Phenotypic characterization of animal genetic resources. FAO Animal Production and Health Guidelines No. 11. Rome. URL: https: / / bit.ly / 2W5FnEv.

FAO/BID (2007). Politicas para la agricultura familiar en América Latina. Oficina regional de la FAO para América Latina y el Caribe. URL: https: / / bit.ly / 2CvcUzO.

Falagan, A. (1988). Caracterización productiva de la raza caprina murciana-granadina en la región de Murcia. Aspectos técnicos y sociales. Inf. téc. 63. Madrid: Instituto Nacional de Investigaciones Agrarias. Ministerio de Agricultura, Pesca y Alimentación, MAPA. Colección Monografías INIA., pág. 103.

Fikru, S. y K. Gebeyew (2015). «Sheep and Goat Production Systems in Degehabur Zone, Eastern Ethiopia: Challenge and Opportunities». En: Advances in Dairy Research 3.2, 134. Online: https: / / bit.ly/2QkZiPa.

Garcia, A. y col. (2010). «Caracterización estructural de los sistemas ganaderos de las dehesas andaluzas». En: Archivos de Zootecnia 59.228, 577-588. Online: https://bit.ly /2DBCYKp.

Gómez, N. (2013). «Caracterización estructural, morfológica y genética de la población de cabras autóctonas de la región Apurímac del Perú». Tesis doctoral. Universidad Autónoma de Barcelona (UAB), Facultad de Veterinaria. Departamento de Ciencia Animal. Online: https: / / bit.ly / 2QJoCgT.

Greenacre, M. (1984). Theory and Applications of Correspondence Analysis. London: Academic Press. 
Guerrero, L. y col. (2012). «Caracterización de los sistemas de producción caprinos presentes en el municipio de Jordán, Santander, Colombia». En: AICA 2, 171-174. Online: https: / / bit.ly / $2 \mathrm{Wh} 6 \mathrm{kFv}$.

Hagos, G. y col. (2016). «On-Farm phenotypic characterization of Begait goat and their production system in Western Zone of Tigray, Ethiopia». En: International Journal of Research and Innovation is Earth Science 3.1, 15-20. Online: https: / / bit.ly / 2Zwi1tE.

Hernández, J. y col. (2011). «Caracterización socioeconómica y productiva de unidades caprinas familiares en la Mixteca Poblana. Redalyc, Sistema de Información Científica, Red de Revistas Científicas de América Latina, el Caribe, España y Portugal». En: Archivos de Zootecnia 60.230, 175-182. Online: https://bit.ly/2Dy2ccN.

Hernández, P. y col. (2013). «Tipificación de los sistemas campesinos de producción de leche del Sur del Estado de México». En: Universidad y Ciencia, Trópico húmedo 29.1, 19-31. Online: https: / / bit.ly/2voDOWB.

Hodges, J. y col. (2014). «Globalisation and the sustainability of farmers, livestock-keepers, pastoralists and fragile habitats». En: Biodiversity 15.23, 109-118. Online: https://bit.ly/35qpCeZ.

Instituto Nacional de Estadística y Censos (2010). Fascículo Santa Elena.

Jarvis, D., C. Padoch y D. Cooper (2011). Manejo de la agrobiodiversidad en los ecosistemas agrícolas.

Lanari, M. R. (2004). «Variación y diferenciación genética y fenotípica de la cabra criolla Neuquina en relación con su sistema rural campesino». Tesis doctoral. Facultad de Ciencias Biológicas. Centro Regional Universitario Bariloche. Universidad Nacional del Comahue. Online: https: / / bit.ly/35o5WZs.

Luque, C. (2011). «Caracterización y evaluación de las razas caprinas autóctonas españolas de orientación cárnica». Tesis Doctoral. Universidad de Córdoba. Departamento de Producción Animal España. Online: https : / / bit . ly / 2DBkKZI.

Macías, A. (2015). «Propuesta de mejoramiento en la gestión de pequeños productores caprinos en Capitanejo, Santander». Doctoral dissertation. Universidad Nacional de Colombia-Bogotá. Online: https:/ / bit.ly/2VjniFX.

Ministerio de Agricultura y Ganadería (2018). Entrega de Nombramiento a los Cabildos Comunales elec- tos para el periodo 2018. Online: https://bit.ly/ 2kFfT3j.

Navarro, M., G. Fernández y R. Perezgrovas (2011). "Characterization of Majorera goat production systems in the Canary Islands. In Bernués A. (ed.), Boutonnet J.P. (ed.), Casasús I. (ed.), Chentouf M. (ed.), Gabiña D. (ed.), Joy M. (ed.), López-Francos A. (ed.), Morand-Fehr P. (ed.), Pacheco F. (ed.). Economic, social and environmental sustainability in sheep and goat production systems. Zaragoza: CIHEAM /FAO/CITADGA. 205-210». En: Options Méditerranéennes: Série A. Séminaires Méditerranéens 100, Online: https://bit.ly/2vjW8A3.

Núñez-Domínguez, R. y col. (2016). «La adaptabilidad de los recursos zoogenéticos Criollos, base para enfrentar los desafíos de la producción animal». En: Archivos de Zootecnia 65.251, 461-468. Online: https:/ / bit.ly/2QPVGEa.

Peña, D. (2002). Análisis de Datos Multivariante. España: Mc Graw-Hill.

Robinson, T. P. y col. (2011). «Global livestock production systems». En: FAO $\mathcal{E}$ ILRI, Online: https:/ / bit.ly/35i8cBf.

Serrano, M. (2010). «Análisis del sistema de producción de cabras con fines lecheros en la región de Libres, Puebla». Tesis de Maestría. México: Colegio de Postgraduados. Online: https: / / bit.ly / 2QkXdD4.

Sistema Nacional de Información (2018). Encuesta de Superficie y Producción Agropecuaria Continua (ESPAC) 2004-2013. URL: https:/ / bit.ly/2lIMHIY.

Torres, P. (2014). «Caracterização e modelagem dos sistemas de produção de caprinos leiteiros». Tesis de mtría. Macaíba/RN - Brasil: Universidade Federal do Rio Grande do Norte. Online: https: //bit.ly/2L0SllS.

Tsegaye, T. (2009). «Characterization of goat production systems and on-farm evaluation of the growth performance of grazing goats supplemented with different protein sources in Metema Woreda, Amhara region, Ethiopia». M.Sc. Thesis, Science in Agriculture (Animal Nutrition). Haramaya University. Department of Animal Science, School of Graduate Studies. Online: https:/ / bit.ly/2UCIkL6.

Umunna, M., O. Olafadehan y A. Arowona (2014). «Small rumiant production and management systems in urban area of Southern Guinea Savanna of Nigeria». En: Asian Journal of Agricul- 
ture and Food Science 2.2, 107-114. Online: https: //bit.ly/2ZIh8hW.

Valerio, D. y col. (2010). «Caracterización Estructural del Sistema Ovino-Caprino de la Región noroeste de República Dominicana». En: Archivos de Zootecnia 59.227, 333-343. Online: https:/ / bit. ly/2QIiPIy.

Vargas, J. y col. (2014). «Caracterización de los sistemas de producción caprina en el municipio de
Aratoca, Santander sobre la cuenca del río Chicamocha». En: AICA 4, 234-236. Online: https: / / bit.ly /2vj794B.

Ward, H. (1963). «Hierarchical Grouping to Optimize an Objective Function». En: Journal of the American Statistical Association 58, 236-244. Online: https://bit.ly/2ZwusFX. 